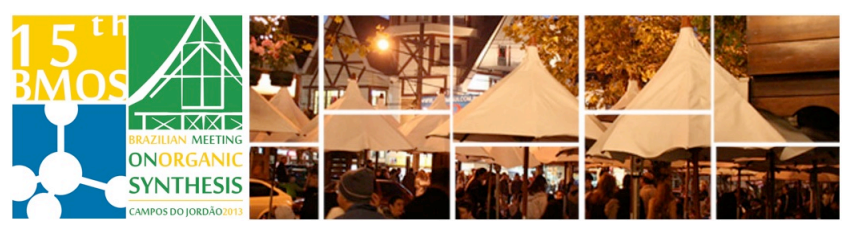

\title{
An expeditious approach to tetrahydroindolizines from Morita- Baylis-Hillman Adducts
}

\section{Bruno V. M. Teodoro, José Tiago M. Correia and Fernando Coelho*}

Laboratory of Synthesis of Natural Products and Drugs, Institute of Chemistry, University of Campinas-

\author{
UNICAMP, P.O.Box 6154, 13083-970, Campinas-SP-Brazil
}

*coelho@iqm.unicamp.br

Keywords: indolizines, Morita-Baylis-Hillman (MBH) Reaction, hydrogenation

\section{INTRODUCTION}

Indolizines are important building blocks for synthesis of biomarkers, drugs and alkaloids that posses the indolizidinic core. This type of heteroaromatic skeleton can be prepared using different approaches. ${ }^{1}$ Recently Morita-BaylisHillman adducts were used as building block for the synthesis of indolizidinic unity ${ }^{2,3}$. However, applications of these molecules remain almost unexplored.

In this communication, we disclosed our preliminary results on a simple strategy to access tetrahydroindolizines and indolizidines by partial or total hydrogenation of indolizines prepared from $\mathrm{MBH}$ adducts.

\section{RESULTS AND DISCUSSION}

The indolizines (3a-f) were prepared from $\mathrm{MBH}$ adducts (1a-f) according to a methodology reported on literature some years ago.

$\stackrel{\mathrm{DABCO},))}{\longrightarrow}$

Scheme 1. Preparation of indolizines from $\mathrm{MBH}$ adducts

Table 1. Indolizines from MBH adducts.

\begin{tabular}{|c|c|c|c|c|}
\hline Entry & Aldehyde & Olefin & $\begin{array}{l}\text { MBH Adduct } \\
\text { (Yield \%) }\end{array}$ & Indolizine (Yield) \\
\hline 1 & & $\pi^{\sigma_{2} \text { we }}$ & $1 a(93)$ & $\begin{array}{l}3 \mathrm{a}(55) \\
3 \text { - }\end{array}$ \\
\hline 2 & & & $1 b(96)$ & $\begin{array}{l}\text { N }>\mathrm{Co}_{2} \mathrm{Et} \\
3 \mathrm{~b}(52)\end{array}$ \\
\hline 3 & & & 1c (93) & $\begin{array}{l}3 c(59) \\
3 c-\mathrm{Co}_{2} \text {-Bu }\end{array}$ \\
\hline 4 & & & $1 d(85)$ & $3 d(39)$ \\
\hline 5 & & & $1 \mathrm{e}(88)$ & $3 e(45)$ \\
\hline
\end{tabular}

With the indolizines in our hands, hydrogenation reactions were performed. Four protocols were tested. First, we decide to reproduce an experiment described previously where the Adam's catalyst $\left(\mathrm{PtO}_{2}\right)$ were used under $1 \mathrm{~atm}$ hydrogen atmosphere. In these cases, we observed partial reduction of indolizines, affording tetrahydroindolizines as unique products in excellent yields (Table 2). The same results can be obtained using $\mathrm{Pd} / \mathrm{C}$ catalyst. However, when $\mathrm{Rh} / \mathrm{C}$ and $\mathrm{Rh} / \mathrm{Al}_{2} \mathrm{O}_{3}$ catalysts were used, no reduction was observed.

$$
\text { } \underset{\mathrm{CH}_{3} \mathrm{OH}, \mathrm{H}_{2}(\mathrm{bar})}{\stackrel{\mathrm{PtO}_{2}(10 \mathrm{~mol} \%)}{\longrightarrow}}
$$

Scheme 2. Preparation of tetrahydroindolizines

Table 2. Tetrahydroindolizines from indolizines

\begin{tabular}{|c|c|c|c|}
\hline Entry & Indolizine & Tetraidroindolizine & $\begin{array}{l}\text { Yield } \\
(\%)\end{array}$ \\
\hline 1 & $3 a$ & $4 a$ & 93 \\
\hline 2 & $3 b$ & $4 b$ & 91 \\
\hline 3 & $3 c$ & $4 c$ & 95 \\
\hline 5 & $3 d$ & $4 d$ & 79 \\
\hline 6 & $3 e$ & $4 e$ & $67^{\mathrm{a}}$ \\
\hline
\end{tabular}
atmosphere.

\section{CONCLUSION}

Our results clearly demonstrated that partial reduction of indolizines can be achieved in excellent yields, at low pressure, using simple reducing agents. Studies focused on partial or total asymmetric hydrogenation using homogeneous catalysts are ongoing in our laboratory.

\section{ACKNOWLEDGEMENTS}

We thank FAPESP and CNPq for grants and financial support.

\section{REFERENCES}

${ }^{1}$ Gadad, A. K.; Mahajanshetti, C. S.; Nimbalkar, S.; Raichurkar, A. Eur. J. Med. Chem. 2000, 35, 853. ${ }^{2}$ Coelho, F.; Almeida, W. P.; Mateus, C. R.;

Feltrin, M.; Costa, A. M. Tetrahedron 2001, 57, 6901. ${ }^{3}$ Rodrigues Jr., M. T.; Gomes, J. C.; Smith, J.; Coelho, F. Tetrahedron Lett. 2010, 51, 4988. 